\title{
Small and Repetitive Axial Strain Reducing the Critical Current in BSCCO/Ag Superconductors
}

\author{
Bennie ten Haken, André Beuink and Herman H. J. ten Kate. \\ Low Temperatures Div., University of Twente, P.O. Box 217, 7500 AE Enschede, The Netherlands
}

\begin{abstract}
The critical current in two types of axially deformed $\mathrm{BSCCO} / \mathrm{Ag}$ tape conductors is investigated. An $I_{c}$ reduction is observed for small axial strains (ranging from 0 to $0.3 \%$ ) with a characteristic slope $d i_{c} / d \varepsilon=-5 \pm 1$ (relative $i_{c}$ change per relative change in length). In the case of an axial compression there is a more pronounced $I_{c}$ reduction. For small axial strains $(<0.3 \%)$ a certain reversible change in $I_{c}$ is observed. This reversible behaviour occurs in combination with an irreversible reduction that increases when the number of strain cycles is increased. The reversible part of the $I_{c}$ change remains for a large number of strain cycles $(>10000)$ and has a similar negative slope for both compressive and tensile strains. It is proposed that the reversible $I_{c}$ change is correlated to a non-hydrostatic lattice deformation. The $I_{c}$ versus strain behaviour is in good agreement with an earlier proposed model.
\end{abstract}

\section{INTRODUCTION}

The critical current of poly-crystalline BSCCO can be reduced significantly due to a certain deformation. This is observed in deformed BSCCO/Ag superconductors. Experiments on $\mathrm{Bi}-2212$ and 2223 conductors showed an irreversible $I_{c}$ reduction for an axial tension and compression and after a transverse compression [1],[2]. The irreversible nature of the $I_{c}$ reductions and the absence of a temperature or field dependence forces to conclude that the $I_{c}$ change is attributed to (micro-)cracks in the poly-crystalline structure.

In this paper a detailed study is presented on the $I_{C}$ in $\mathrm{BSCCO} / \mathrm{Ag}$ superconductors, when deformed in the strain range from -0.3 to $0.3 \%$. The influence of repetitive tensile and compressive axial strains, with a peak value around $0.2 \%$, is investigated. Special attention is paid to the reversibility of the $I_{c}$ change after multiple strain variations. The results are evaluated in relation to the descriptive model that is presented earlier [2].

\section{SAMPLE MATERIAL}

The deformation experiments are made with two prototype superconductors recently obtained from different manufacturers. Both tapes are produced with the "powder-in-tube" process. The relevant characteristics are summarised in Table 1 . These conductors have a good $J_{c}$, but the highest current density is measured in conductor-B. This higher $J_{c}$ correlates to a higher degree of compaction of the filaments. An optical analysis of the tape cross-section shows that the filaments in conductor-A contain significantly more voids (about $30 \%)$ than those in conductor-B $(10-20 \%)$.

Manuscript received August 24, 1996
TABLE I

THE TWO BI-2223/AG TAPES FROM DIFFERENT MANUFACTURERS

\begin{tabular}{rcc}
\hline Conductor & A & B \\
\hline Size $[$ mm $\times m m]$ & $3.4 \times 0.25$ & $4.1 \times 0.30$ \\
No. filaments & 49 & 85 \\
Ag $:$ S.C. & $4: 1$ & $3: 1$ \\
$J_{c}$ (non-Ag @ $\left.77 \mathrm{~K}\right)\left[\mathrm{A} / \mathrm{mm}^{2}\right]$ & 75 & 130 \\
\hline
\end{tabular}

\section{STATIC AXIAL STRAIN}

The characterisation of the critical current as a function of the axial strain is made with a "bending-spring", where the conductor is tightly connected to a thick substrate [3]. This set-up enables the determination of $I_{c}$ in a strain range from $1 \%$ to $+1 \%$ uni-axial strain. Consequently it requires at least two pieces of sample material in order to investigate both the compressive and tensile strain regime. A second feature is that the thermal compression in the last part of the cooling phase, from soldering at $500 \mathrm{~K}$ down to $77 \mathrm{~K}$, is fixed and mainly determined by the thermal contraction of the bending spring (brass).

\section{A. Tensile Strain}

The reduction of the $I_{c}$ due to tensile strain is similar to that observed in many other BSCCO/Ag conductors. The results for the two conductors are presented in figure 1. The strain state after cooling to $77 \mathrm{~K}$ is defined as zero.

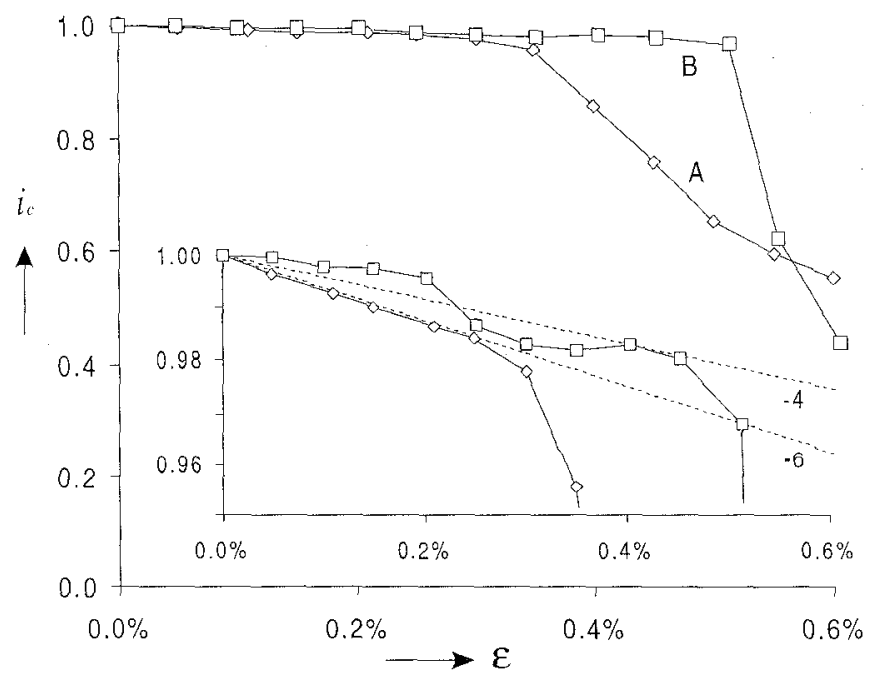

Fig. 1. The reduction of the normalised critical current $i_{c}=I_{c}(\varepsilon) / I_{C}(0)$ as a function of the tensile strain (including a magnified part of the curve). 
Up to a certain limit of strain $(0.45 \%$ for $\mathrm{A}$ and $0.30 \%$ for B) the $I_{c}$ remains almost constant. For a larger strain the current reduces drastically to below 0.6 of its original value at $0.6 \%$ strain. The $I_{c}$ reduction, where the current reduction is small, is presented also in figure 1 . The $I_{c}$ reduction in this regime can satisfactory be described with a constant slope in the normalised critical current of $d i_{c} / d \varepsilon=-5 \pm 1$. This strain performance of the two tapes is in good agreement with earlier results on other $\mathrm{Bi}-2223 / \mathrm{Ag}$ tapes [2].

\section{B. Compressive Strain}

The $I_{c}$. reduction in axially compressed conductors can be investigated very well with the bending spring. The results are depicted in figure 2. A regular $I_{c}$ reduction is observed that can be described with a constant relative change of $d i_{c} / d \varepsilon=20 \pm 2$. Again, this is in good agreement with the lowest values of earlier results on axially compressed $\mathrm{Bi}$ 2223/Ag tape [2].

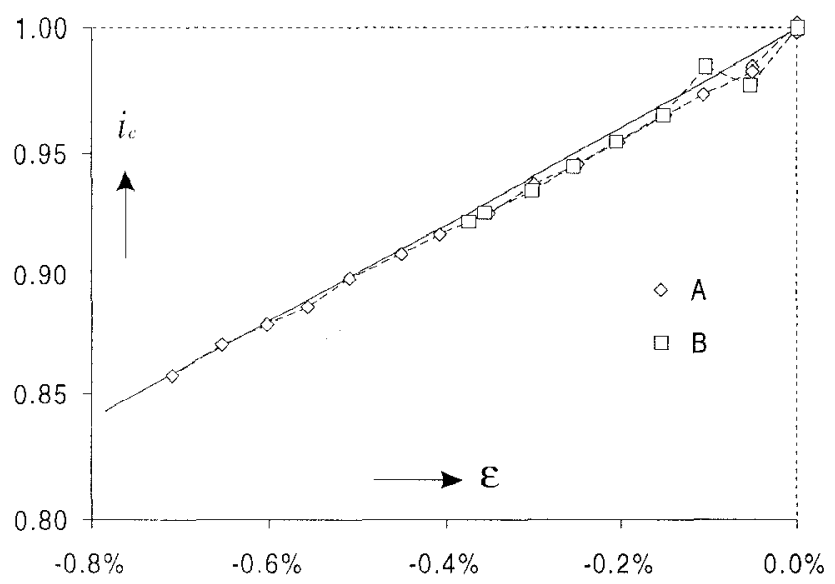

Fig. 2. The critical current reduction due to a compressive axial strain in conductors $\mathrm{A}$ and $\mathrm{B}$.

\section{Comparison with the $I_{c^{-}}-$strain model}

The observed $I_{c}$ versus strain relation in combination with the irreversible nature of the $I_{c}$ reduction, lcad to the proposal of a descriptive model [1]. This model assumes an $I_{c}$ in the strain-free state that is slightly above the $I_{c}$ measured at the beginning of the strain experiment, as depicted in figure 3 .

Due to the thermal compression of the BSCCO, the critical current is already reduced by a certain factor at the beginning of the strain experiment. In fact the strain history already starts at about $830^{\circ} \mathrm{C}$ just after the solidification of the BSCCO. When the conductor is then compressed the $I_{c}$ decreases further, but when a tension is applied the $I_{c}$ deviates from its original line. The irreversible nature of the $I_{c}$ reduction limits the $I_{c}$ to the value present in this strain state. Finally for a larger tension, when the tensile strain counteracts the thermal compression, a strong $I_{c}$ reduction occurs.

The differences in the $I_{c}$ values between the different manufacturers and the variations within a single production batch make it at present not possible to compare the $I_{c}$ between conductor samples with a different thermal contrac- tion, either by comparing the $I_{c}$ between different types of conductors or by investigating the influence of different sample-holder materials.

As mentioned before, the results found with these two conductors are in good agreement with earlier results on $\mathrm{Bi}$ $2223 / \mathrm{Ag}$ and $\mathrm{Bi}-2212 / \mathrm{Ag}$ conductors and the model that is proposed to describe the behaviour. The back-bone of this description for the strain induced $I_{c}$ reduction is the irreversible nature of the $I_{c}$ reductions. To verify this the reversibility of the $I_{c}$ changes is studied in detail. Cyclic strains are applied in the strain range around the strain state after cooling down to $77 \mathrm{~K}$.

\section{THE REVERSIBILITY OF $I_{C}$ FOR SMALL STRAINS}

The cyclic strain experiments are made on a slightly modified bending spring. The tape is soldered with Indium at $500 \mathrm{~K}$ on a brass substrate, $3 \mathrm{~mm}$ thick and $15 \mathrm{~mm}$ wide. The tape and the substrate are bend over a $25 \mathrm{~mm}$ long section. Outside the deformed section is a $5 \mathrm{~mm}$ zone at each side where the substrate is $5 \mathrm{~mm}$ thick and the deformation is much smaller. The strain in the sample is determined by measuring the deformation of the substrate adjacent to the tape. The $I_{c}$ is determined at a level of $10^{-4} \mathrm{~V} / \mathrm{m}$ with a reproducibility of about $0.3 \%$.

As mentioned before the strain induced $I_{c}$ reductions in the earlier experiments on the bending spring appeared to be completely irreversible. A recovery of the $I_{c}$ when the strain is relieved, was never observed so far. The deformation experiments on conductor-A show a certain recovery of the $I_{c}$ when the strain is relieved. A typical example of this behaviour is presented in figure 4 . The first deformation to $0.28 \%$ strain results in an $I_{c}$ reduction to 0.985 of the initial value. When the strain is reduced back to $0 \%$ strain, then the $I_{c}$ recovers (partially) to 0.989 and the next $I_{c}$ at $0.28 \%$ strain is at 0.980 . In the following strain cycles this process is repeated, the $I_{c}$ at $0 \%$ remains at an approximately $1 \%$ higher value than at $0.28 \%$ strain. This process is not perfectly reversible; after 15 cycles the $i_{c}$ at $0 \%$ strain is reduced to 0.980 .

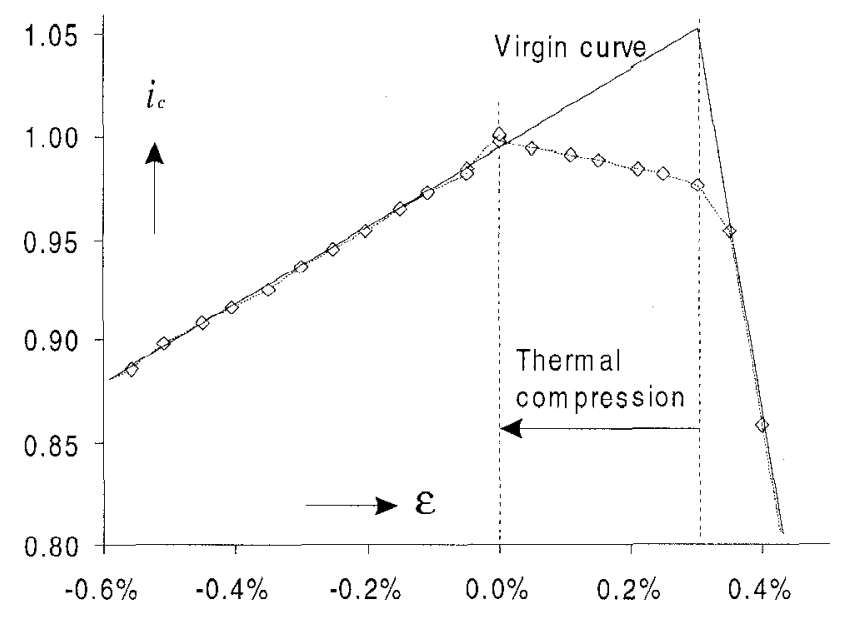

Fig. 3. A comparison between the $i_{c}$ versus strain in sample A and the descriptive model for the $I_{c}$ reduction that defines the virgin curve. 


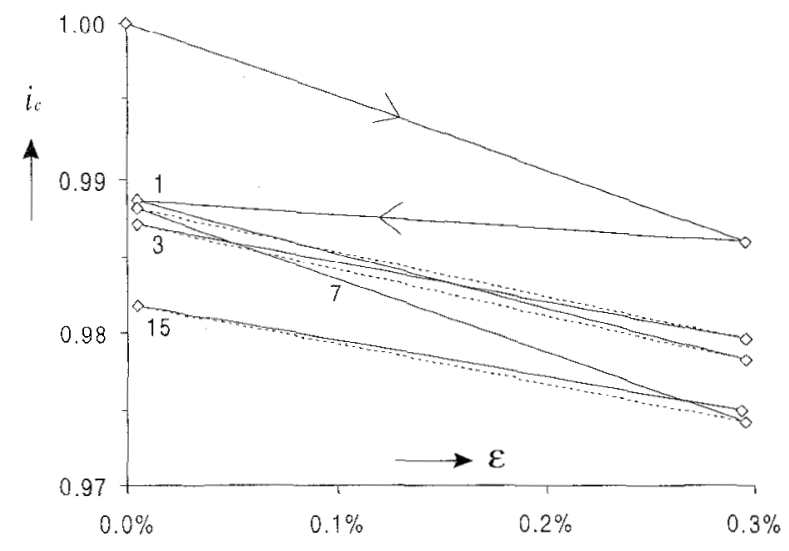

Fig. 4. The $I_{c}$ versus strain in sample A for a cyclic deformation between 0 and $0.28 \%$ axial strain. The solid and dotted lines follow the measuring sequence represented by the numbers too. A solid line indicates two sequential $l_{i}$ measurements and the dotted line is used when one or more strain cycles are skipped.

A very interesting result is obtained when the effect of cyclic strain is compared between the compressive and tensile strain regimes in figure 5 . The first time a compressive strain is applied the $i_{c}$ reduces with a slope of about $d i_{c} / d \varepsilon=20$ to a value of 0.938 . When the strain is relieved to the initial value then the $i_{i}$ reduces further to 0.930 , with a negative slope similar to $d i_{c} / d \varepsilon=-5$. When the number of cycles is increased this negative slope $d i_{c} / d \varepsilon$ remains, when relieving the strain, similar to what happens after multiple cycles in the tensile regime. When increasing the number of strain cycles the $I_{c}$ (at constant strain) further reduces step by step, clearly indicating the irreversible behaviour in the $I_{c}$ reduction.

The $I_{c}$ reductions shown for conductor-A, also occur in a similar way in conductor-B. This behaviour supports the proposed description for the $I_{c}$ of axially strained conductors that is discussed in the previous section. The first compression applied to the conductor reduces the critical current immediately and irreversibly. Tensile strains over a very limited strain regime can show a (partially) reversible $I_{c}$ change with a small slope of typically $d i_{c} / d \varepsilon=-5$.

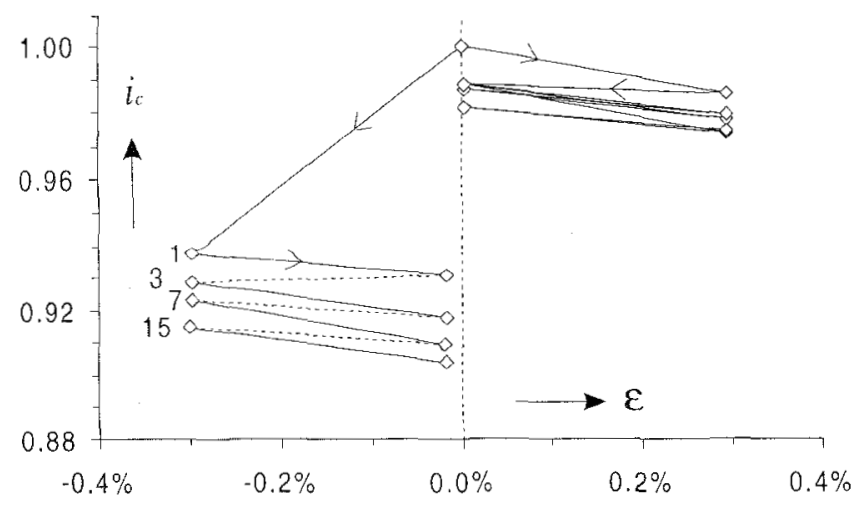

Fig. 5. The $I_{c}$ versus strain in two samples of conductor A. First a cyclic deformation between 0 and $0.28 \%$ axial strain and then between 0 and $-0.28 \%$ strain. The solid and dotted line follows the measuring sequence. The solid lines indicate two sequential $I_{i}$ : measurements and a dotted line is used when one or more strain cycles are skipped.

\section{A LARGE NUMBER OF STRAIN CYCLES}

For technical applications it is important to see the effect of a large number of strain cycles. Axial deformations due to the thermal contraction between room and operating temperature may occur a large number of times during the life time of a cryogenic system (typically 10 to $100 \times$ ). Additional sources of deformation, as Lorentz forces and external forces, may occur much more often $(>1000 \times)$. The (partially) reversible nature of the $I_{c}$ reduction, therefor justifies an investigation of cyclic deformed conductors.

The influence of a cyclic strain on the $I_{c}$ of conductor-A is depicted in figure 6 . Basically it shows that the $I_{c}$ reduces with the number of strain cycles. The difference between the neutral and the elongated state remains constant, within the experimental accuracy, for a large number of cycles of up to at least $10^{4}$. A second observation is that the irreversible part of the $I_{c}$ reduction decreases faster for a larger strain peak value. This reduction may continue, but a certain saturation of the $I_{c}$ at a constant level is more likely.

After the first compressive cycle the $I_{c}$ remains at a higher value than in the tensile strain-state. This shows that the reversible part of the $I_{c}$. change exists and remains negative, for a very large number of cycles. The first compression to $-0.28 \%$ can be considered as a starting point in the cyclic strain experiment. The $I_{c}$ change form this point to a large number of cylces $(0.5$ to 1000$)$ is about $5 \%$, which is similar to the reduction that is observed after a comparable amount of tensile strain cycles.

The reduction of the critical current in conductor-B after multiple strain cycles is presented in figure 7. Again a compressive cycle is compared with a tensile cycle. The behaviour is similar as in conductor-A. The irreversible part of the $I_{c}$. reduction saturates after a large number of cycles. The permanent $I_{\mathrm{c}}$ reduction after $10^{4}$ cycles of $0.2 \%$ tension is $1 \%$. A compressive strain of $-0.21 \%$ initially reduces the $I_{t}$ with nearly $4 \%$, and when this strain is applied multiple times there occurs an additional reduction up to $1 \%$.

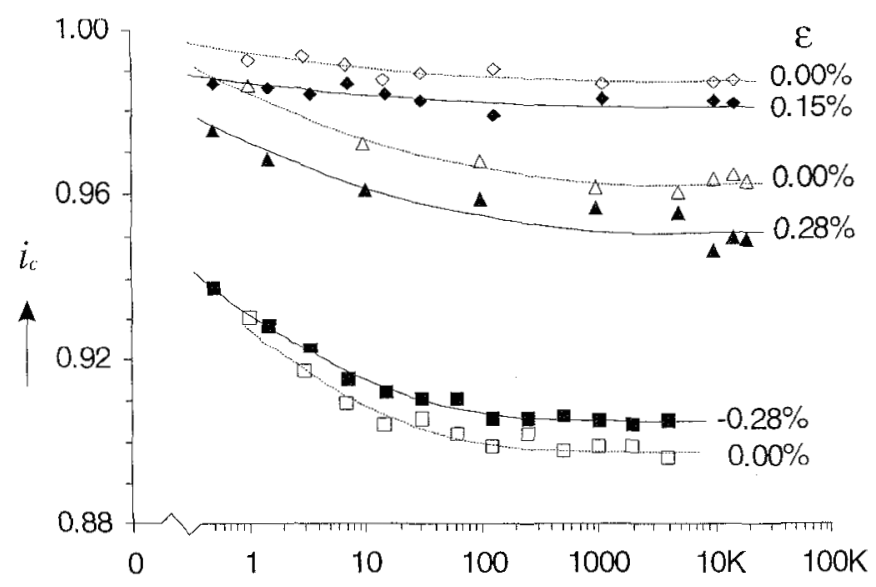

Fig. 6 . The $I_{c}$ versus the number of times strain cycles for three deformation states in conductor-A: 0 to $0.15 \%, 0$ to $0.28 \%$ and 0 . to $-0.28 \%$ strain. The solid lines serve as a guide to the eye. The $I_{c}$ is normalised to the $I_{c}$ after cooling down to $77 \mathrm{~K}$ with a non-deformed bending spring (strain $=0$ and cycle $=0$ ). The first tension or compression is numbered as cycle $=0.5$. 


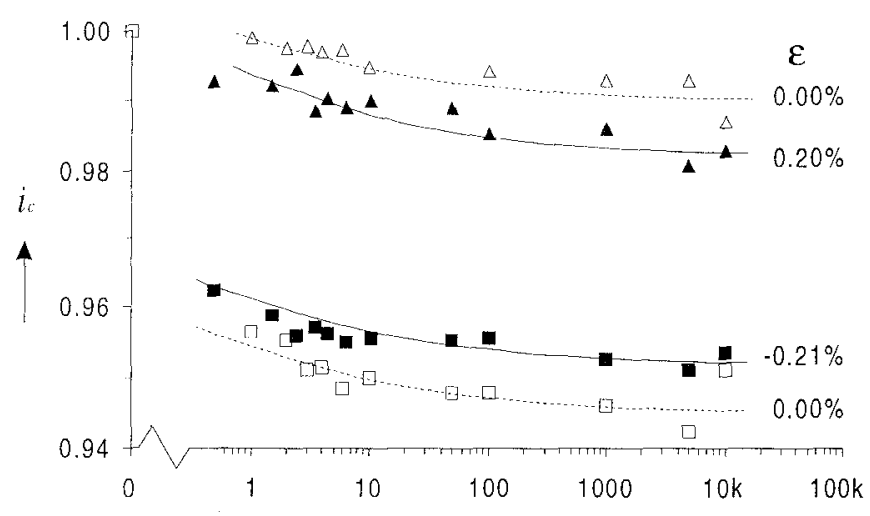

Fig. 7. The $I_{t}$ versus the number of strain cycles in conductor $\mathrm{B}$, for two strain cycles $(0$ to $0.2 \%=$ tensile and 0 to $-0.21 \%=$ compressive $)$.

\section{THE MICROSTRUCTURE IN DEFORMED BSCCO}

As mentioned above the model for the $I_{c}$ in axially strained conductors, that is based on an irreversible $I_{c}$ reduction due to compression, appears to be an adequate description. A new element observed here is the (partially) reversible $I_{c}$ change for small tensile strains. This behaviour is an indication for a certain intrinsic dependence of the $I_{c}$ on the strain state of the material. To our knowledge it is also the first experimental evidence for such an intrinsic $J_{c}(\varepsilon)$ relation in poly-crystalline BSCCO.

The occurrence of reversible lattice deformations in polycrystalline $\mathrm{Bi}-2212$ is recently observed by $\mathrm{X}$-ray diffraction. The lattice deformations are reversible in a very limited strain range of $0.2 \%$ [4]. Uni-axial stress and strain experiments on $\mathrm{Bi}-2212$ whiskers show a reduction in $T_{c}$, when the $a$ - or $b$-axis is clongated and the $c$-axis is compressed [5],[6]. Based on the similar $I_{c}(\varepsilon)$ behaviour it is expected that deformed Bi-2212 and 2223 behave essentially the same. When the observations of the lattice deformation and $I_{c}(\varepsilon)$ in the reversible regime are combined it seems justified to conclude that a slope of $d i_{d} / d \varepsilon=-5$ in the tensile strain regime is determined by the intrinsic properties $d J_{c} / d \varepsilon$ of the grains for the major part.

The irreversible $I_{c}$ reduction that is observed when a certain deformation is applied for the first time, is expected to correlate with the breaking of grain-boundaries in the polycrystalline system. The observed irreversible $I_{c}$ reduction is then determined by a statistical distribution in the strain tolerances of the grain boundaries. The mechanism should also determine the difference between the $I_{c}$ reduction due to an initial tensile or compressive deformation. This process of damaging grain boundaries is also expected to explain the irreversible part of the $I_{c}$, reduction that occurs when a strain cycle is passed multiple times.

\section{CONCLUSIONS}

1 - The static $I_{c}$ versus axial strain behaviour of two different $\mathrm{Bi}-2223 / \mathrm{Ag}$ multi-filamentary conductors is determined and found to be in good agreement with previous results on Bi-2212 and 2223 conductors. Three different strain regimes are distinguished and described by a model for the strain dependence of $I_{c}$ in BSCCO/Ag conductors

2 - A partially reversible $I_{c}$ change is observed when a $\mathrm{BSCCO} / \mathrm{Ag}$ conductor is subjected to a tensile strain. The reversible part of this $I_{c}$ change is believed to be an intrinsic property of the poly-crystaline Bi-2223. Assuming a similar mechanical behaviour in $\mathrm{Bi}-2212$ and 2223, it is proposed that the reversible $I_{c}$ change is correlated to a non-hydrostatic lattice deformation, similar to the $T_{c}$ reduction that is obscrved when the $a$ - or $b$-axis is elongated in a single crystal of Bi-2212.

3 - The irreversible $I_{c}$ reduction due to a large number of strain cycles is investigated for tensile and compressive strain cycles. The experimental results suggest a saturation of the $i_{c}$ after a large number of cycles at a level of 0.99 and 0.95 for strain cycles to 0.15 and $0.28 \%$ strain respectively.

4 - The descriptive model for the irreversible $I_{c}$ reductions in $\mathrm{BSCCO} / \mathrm{Ag}$ conductors is confirmed by static and cyclic strain experiments performed on the two conductors. An important proof for the model is the $I_{c}$ change due to multiple strain cycles. The influence of a compressive strain cycle on $I_{c}$ can be described as a tensile cycle with an enlarged initial compression.

\section{REFERENCES}

[1] B. ten Haken, H.J. Schuver and H.H.J. ten Kate, "Descriptive model for the critical current as a function of axial strain in Bi-2212/Ag wires", IEEE Trans. Mag., 32 No 4, 1996, p. 2720.

[2] B. ten Haken, A. Godeke, H.J. Schuver and H.H.J. ten Kate, "Strain reduced critical current in $\mathrm{Bi}-2223 / \mathrm{Ag}$ superconductors under axial tension and compression", Adv. Cryo. Eng. 42, 1996 (in print).

[3] B. ten Haken, R.B. Wilson, H.H.J. ten Kate and J. Tenbrink, "Critical current reduction due to strain in Bi-2212 wires", in: "Applied superconductivity", editor: H.C. Freyhardt, DGM Informationsgesellschaft mbH, Germany, 1993, p. B235.

[4] B. ten Haken, and H.H.J. ten Kate, "Lattice deformation in an axially strained $\mathrm{BiSrCaCuO} / \mathrm{Ag}$ tape investigated by $\mathrm{X}$-ray diffraction", Physica C, 270, 1996, p. 21.

[5] X. F. Chen, G. X. Tessema and M.J. Skove, Effect of elastic stress on the resistivity and $T_{c}$ of $(\mathrm{Bi}, \mathrm{Pb})_{2} \mathrm{Sr}_{2} \mathrm{CaCu}_{11} \mathrm{O}_{\mathrm{x}}$ ", Physica C 181, 1991, p. 340 .

[6] N. Watanabe et. al., "Effect of Hydrostatic and Uniaxial stress on $T_{c}$ for single Crystals of $\mathrm{Bi}_{2} \mathrm{Sr}_{2} \mathrm{CaCu}_{2} \mathrm{O}_{\mathrm{x}}$ ", Physica C 235-240, 1994, p. 1309. 